\title{
Homogenization of Flow Equations Using the MHD Equation: Numerical Validation
}

\author{
LENORMAND Roland, Institut Français du Pétrole \\ THIELE Marco R., Stanford University
}

Paper presented at the 5th European Conference on the Mathematics of Oil Recovery, Leoben, Austria,3-6 Sept. 1996

\begin{abstract}
Homogenized equations capture flow mechanisms at a sub-gridblock scale and allow for more accurate transport equations to be implemented in numerical simulators. Homogenized equations can also improve the calculation of pseudofunctions like pseudo relative permeabilities and alpha factors. In this study we validate a new form of a homogenized equation previously derived by Lenormand using fine-grid numerical simulations.
\end{abstract}

The MHD-equation accounts for permeability heterogeneities through the dispersion coefficient $D$ and the heterogeneity factor $\mathbf{H}$. The viscous instability is characterized by an apparent viscosity ratio $\mathbf{M}$. The advantage of the MHD-equation is its ability to predict the efficiency of a displacement with viscous effects by using values for the parameters $D$ and $H$ derived from the simpler unit-viscosity-ratio displacement.

In this paper, we compare the results predicted by the MHD-equation to two-dimensional, fine-grid numerical results obtained using a streamtube and a higher-order finite difference simulator.

\section{INTRODUCTION}

Powerful geostatistical algorithms (Deutsch and Journel, 1992) conditioned to $\mathrm{log}$ and core measurements make it possible to generate geological images with a pixel resolution for permeability and porosity on the orders of centimeters. Interwell distances and field dimensions, on the other hand, are on the order of hundreds of meters and kilometers respectively. Field-scale numerical modeling of fluid flow using fine-scale information would therefore result on the order of $10^{12}$ grid blocks which is well beyond current computational resources. As a result, simulations have to be performed on larger grid blocks with average properties derived from the underlying fine-grid information. One approach consists in using upscaling algorithms to homogeneize the permeability heterogeneities, either for one-phase or two-phase flows (King 1989; Pickup et al. 1995; Durlovsky 1991; Kossack et al., 1990). On the other hand, improved transport equations have been derived to account for viscous fingering in homogeneous and hererogeneous media. (Koval 1963, Todd and Longstaff 1972, Fayers 1988, Blunt and Christie, 1991; Gorell 1992, Sorbie et al. 1992, Fayers et al., 1991, 1992). A stochastic approach for deriving a homogenized flow equation accounting for both fingering and heterogeneities has recently been presented by Lenormand $(1995,1996)$. In this paper, we validate the approach proposed by Lenormand numerically by considering first-contact miscible displacements in 20 heterogeneous fields with 25,000 gridblocks and varying statistical properties.

The principal idea underlying the homogenized flow model is to find an equation that can approximate the displacement of one fluid by another in the principal flow direction under the following assumptions:

1) At the small scale, the interface between the fluids is assumed to be sharp, and there is no mixing or capillary spreading. This assumption corresponds to the ideal case of immiscible fluids with negligible capillary pressure and a flux function of the form $\mathrm{f}=\mathrm{S}$.

2) The injection is continuous at the inlet (step injection).

3) Gravity effects are negligible.

\section{THE "MHD" EOUATION,}

The result of the stochastic calculation presented by Lenormand $(1995,1996)$ is a general transport equation with a nonlinear first-order convective term and nonlinear second-order dispersive term.

$$
\begin{gathered}
\frac{\partial S}{\partial t}+u \frac{\partial f}{\partial x}=D \frac{\partial}{\partial x}\left(\frac{f}{S} \frac{\partial S}{\partial x}\right) \\
f=\frac{S}{S+(1-S) / H M^{*}}
\end{gathered}
$$

Here $S$ is the saturation/concentration, $f$ is the flux function, $u$ is the mean front velocity, $D$ is the 
dispersion coefficient, $\mathrm{H}$ is the heterogeneity factor, and $M^{*}$ is the effective viscosity ratio, which is assumed to be directly related to the real viscosity ratio $M$.

$$
M=\frac{\mu(S=0)}{\mu(S=1)}
$$

The MHD model assumes that the stochastic properties of the permeability field can be lumped into the heterogeneity factor $\mathrm{H}$ and the macrodispersion coefficient $\mathrm{D}$. The left hand term of the MHD equation is similar to the equation proposed by Koval (1963) to describe viscous instability in homogeneous media $(H=1)$ or slightly heterogeneous $(H>1)$. The MHD equation can be seen as an extension of Koval's model to strongly heterogeneous media.

The usefulness of the MHD equation stems from the ability to predict the spreading (and consequently the pseudo relative permeabilities) of non-unit viscosity ratio displacement when the two parameters, $H$ and $D$, are known. These parameters are derived either by a stochastic calculation from the properties of the permeability field or by simulation of a unit mobility $(\mathrm{M}=1)$ displacement.

The purpose of this study is to validate this equation by using numerical simulations with a level of numerical dispersion that is sufficiently low so as not to mask any dispersive/convective property of the underlying permeability field.

We validate the MHD equation as follows:

1) Generate 2 dimensional log normal permeability distributions with given stochastic properties (correlation length and variance of $\log \mathrm{K}$ ) using GSLIB (Deutsch and Journel, 1992). All permeability fields are assumed to have 25,000 blocks ( $\mathrm{xx}=250$; ny=100).

2) Perform fluid flow simulations for $M=1$.

3) Determine parameters $H$ and $D$ for the various permeability fields by fitting the front spreading with the spreading predicted by the MHD equation.

4) Perform fluid flow simulations for $M>1$.

5) Determine the two parameters D and HM*

6) Validate the relationship between the effective viscosity ratio $M^{*}$ and the real mobility ratio $M$, using Koval's model.

\section{CHARACTERIZATION OF FRONT SPREADING}

There are several methods to characterize the width of a front (for instance the distance between saturations $S=0.1$ and $S=0.9$ ). We use a more general definition, which accounts for the average properties and smoothes the local fluctuations of the front. We first calculate the average saturation in the $x$ direction by summing the amount of injected fluid long a slice of thickness $\mathrm{dx}$. The "mass" $d m$ of interface in the $x$ direction between $x$ and $x+d x$ is proportional to $d S$ (Fig. 1).

$$
\mathrm{dm} \propto \mathrm{dS}=\frac{\partial S}{\partial \mathrm{x}} \mathrm{dx}
$$

The average front position is the first moment of the mass of interface

$$
x_{f}=\int_{0}^{L} x d m / \int_{0}^{L} d m
$$

Assuming that entrance saturation is 1 and $S(L)=0$ (before breakthrough) leads to

$$
x=-\int_{0}^{L} x \frac{\partial S}{\partial x} d x
$$

Under the same assumptions, the variance of the front is

$$
\sigma_{x}^{2}=-\int_{0}^{L}\left(x-x_{f}\right)^{2} \frac{\partial S}{\partial x} d x
$$

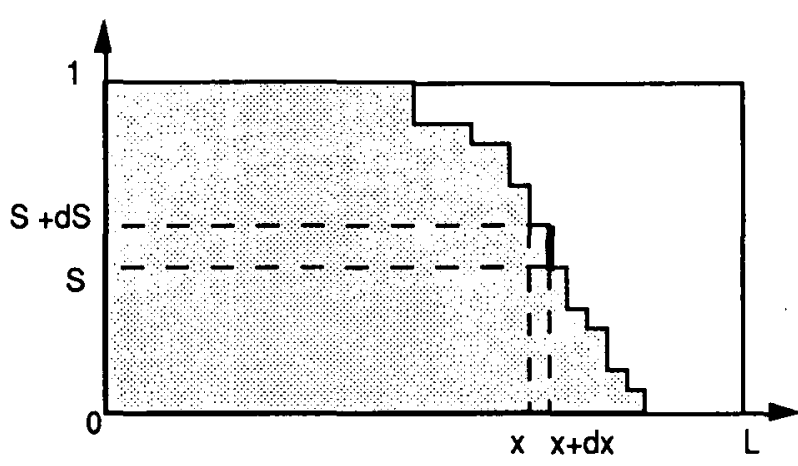

Fig. 1 - Principle of the characterization of front spreading

Note that the calculation of the spatial moments is limited at breakthrough. This limitation does not exist for the temporal moment (using the flux instead of saturation) and thus it is possible to determine the spreading well beyond breakthrough.

\section{MOMENTS OF THE MHD EOUATION}

The spatial variance $\sigma_{x}^{2}$ can be calculated directly from the MHD equation by multiplying each member by $x^{2}$ and integrating with respect to space. Several integrations per part lead to an analytical equation for the variance (before breakthrough). The calculation is done for $M=1$ in Appendix 1. In dimensionless form (length $\mathrm{L}$ of the medium and time to fill one pore volume as references), the result is the following

$$
\sigma_{x}^{2}=\frac{(H-1)^{2}}{3 H} t^{2}+2 D \frac{H \ln (H)}{H-1} t
$$

The variance is the sum of a convective and a dispersive contribution. For the limiting dispersive case $(\mathrm{H}=1)$, the variance is given by the standard equation $\sigma_{x}^{2}=2 \mathrm{Dt}$. 

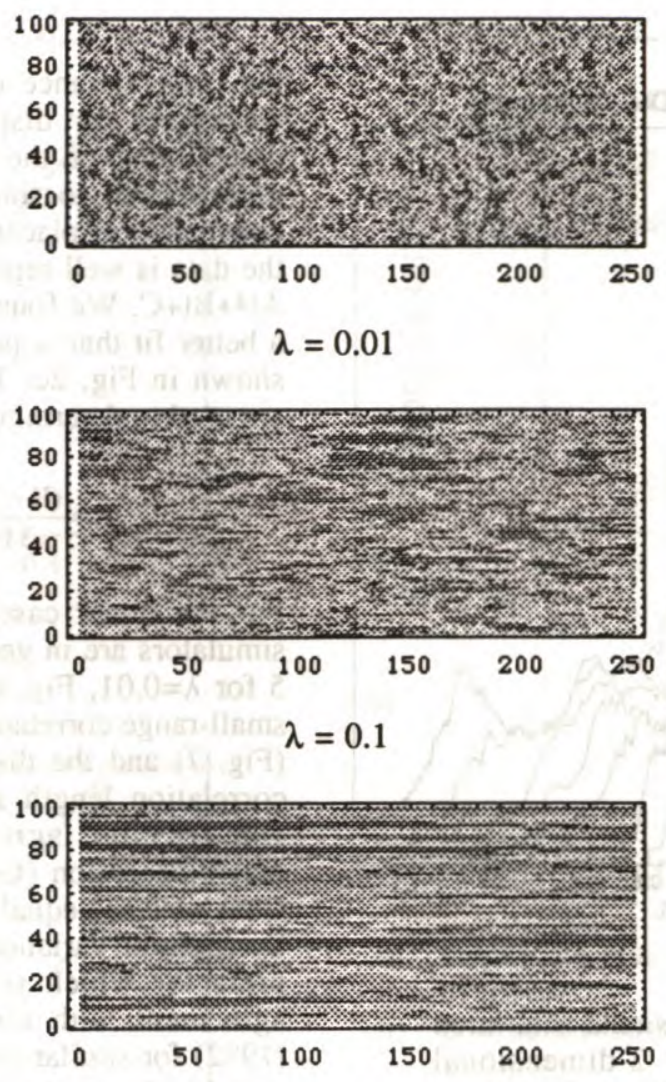

$\lambda=1$

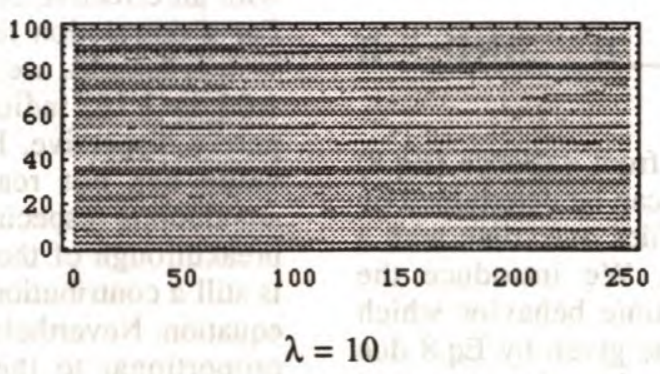

Fig. 2 - Examples of permeability fields used for the simulations $(\sigma(\log K)=1)$

\section{PERMEABILITY FIELDS}

The permeability fields were created by using GSLIB (Deutch and Journel, 1992) and contain 250×100 gridblocks. We used exponential variograms and a log normal permeability distribution for all cases. We considered five different correlation lengths: $\lambda=0.001$, $0.01,0.1,1,10$ and four values for $\sigma(\operatorname{logK}): 0.1,0.5,1$ and 2 . Using these 20 permeability fields we were able to cover the range from completely random media to almost perfectly layered media with varying degree of permeability contrasts. Example permeability fields for $\sigma(\log K)=1$ are shown in Fig.2.

\section{NUMERICAL SIMULATIONS}

The numerical simulations for this work were performed using a finite-difference code with a fluxcorrected transport formulation to minimize numerical diffusion (Christie 1989) and a recently proposed approach using streamtubes and streamlines (Thiele et al. 1995). All simulations were done assuming no physical dispersion. The streamtube approach has been shown to be completely free of numerical dispersion and exact for $M=1$ displacements, since in this special case the streamtubes remain constant in time. For the $M>1$ simulations we used the finite-difference code. To generate the average one-dimensional profiles, the twodimensional saturation distribution was averaged arithmetically over the number of blocks in the ydirection (100) at the center of each block in the $\mathrm{x}$ direction (250) at 10 time intervals. Fig.3 shows an example of the resulting one-dimensional profiles from the two-dimensional saturation maps. 


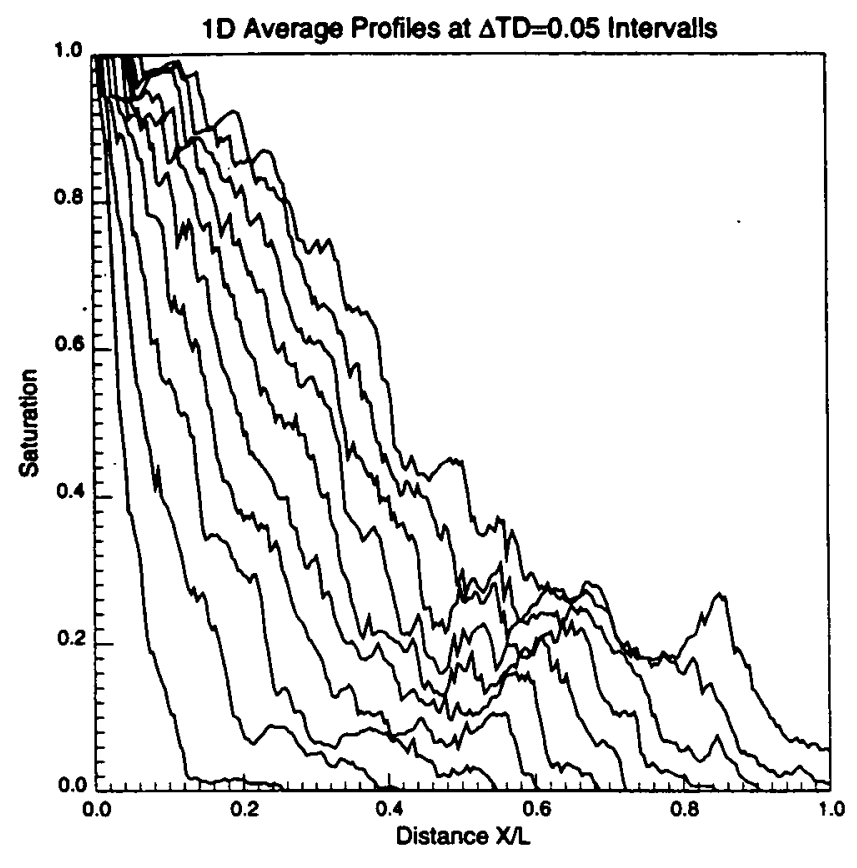

Fig. 3 - Example of 1-dimensional saturation profiles derived from the 2-dimensional saturation maps $(M=2 ; \sigma(\operatorname{logK})=1, \lambda=0.1)$.

\section{DETERMINATION OF D AND H PARAMETERS}

The analytical expression for the front variance (Eq.8) suggests that parameters $\mathrm{H}$ and $\mathrm{D}$ can be determined by a least square fit of the front profiles over time with a function of the form $A t^{2}+B t+C$. We introduce the constant $\mathbf{C}$ to account for early time behavior which may differ from the theoretical one given by Eq.8 due to the finite number of blocks used in the numerical simulations. The constant $C$ is always small compared to A or B.

\section{RESULTS}

We have performed displacement simulations for the tracer case $(M=1)$ using both the streamtube method and the FCT finite-difference simulator. For cases with $M>1$ we used only the FCT finite-difference simulator. In all cases the front variance is calculated from $1 \mathrm{D}$ profiles before breakthrough.

Figure 4 shows three different examples of front spreading: dispersive spreading, convective spreading, and mixed spreading. Time and front variance in Fig.4 are in dimensionless form given by $t^{*}=u t / L$ and

$$
\sigma_{x}^{2}=\frac{(H-1)^{2}}{3 H} t^{2}+2 D^{*} \frac{H \ln (H)}{H-1} t
$$

where $D^{*}$ is the dimensionless dispersion coefficient given by $\mathrm{D} / \mathrm{uL}$. For small correlation lengths $(\lambda=0.01)$, the front variance is proportional to time (Fig 4a), as we expect for dispersive displacements. Long-range correlations, on the other hand, lead to a front variance proportional to time squared (Fig. 4b), indicating a convective displacement. For the crossover regimes, all the data is well represented by a 2 nd order polynomial, $A t^{2}+B t+C$. We found the 2 nd order polynomial to give a better fit than a power law model. Two examples are shown in Fig. 2c. The values for parameters A and B are used to determine $\mathrm{H}$ and $\mathrm{D}$ by solving

$$
A=\frac{(H-1)^{2}}{3 H} \text { and } B=2 D^{*} \frac{H \ln (H)}{H-1}
$$

For the tracer case $(M=1)$, the results from the two simulators are in good agreement (see for instance Fig. 5 for $\lambda=0.01$, Fig. 6 for $\lambda=10$ and Fig. 7 and 8). For small-range correlations, the value of $\mathbf{H}$ is close to unity (Fig. 7) and the dispersivity $\mathrm{D}^{*}$ is a function of both correlation length and permeability contrast (Fig. 8). Our results agree with Gelhar' definition of macrodispersion (Gelhar et al., 1983) which predicts a front variance equal to the product of correlation length by squared variance of logK (Fig. 5). except for the prefactor which is not unity but equal to 0.38 . in agreement with the factor 0.32 obtained by Gorell (1992) for similar simulations. Since there are only 250 grid blocks in the $\mathrm{x}$-direction, the dispersivity for all $\lambda=0.001$ represents the dispersivity for a random field with an effective correlation length of $\lambda=0.004=1 / 250$. For a correlation length equal to $\lambda=0.01$ (1/00 of the system length), we found values for $\mathrm{H}$ in the range of 1.06 to 1.16 , indicating that the displacement is not purely dispersive. For $\lambda=10$, on the other hand, the $H$ value has not reach its asymptotic limit in most simulations (especially for $\sigma(\operatorname{logK})=2$ ) due to the early breakthrough of the simulations. That means that there is still a contribution of the dispersive term in the MHD equation. Nevertheless, we found the coefficient $A$ to be proportional to the variance of $\log (K)$ and $H$ well described by a power law of the type (Fig.6)

$$
H=2.5 \sigma(\log K)^{0.63}
$$

For non-unity viscosity ratios the front variance is still well fitted by a 2 nd order polynomial (Fig. 4c). The convective factor $A$, can now be used to calculate the product $\mathrm{HM}^{*}$ (using $\mathrm{Eq}$. 8). Using the $\mathrm{H}$ value from the tracer case, allows to determine $M^{*}$ as a function of the true viscosity ratio $M$.

$$
M *=0.2 M+0.8
$$

or a power law

$$
M^{*}=M^{0.4}
$$




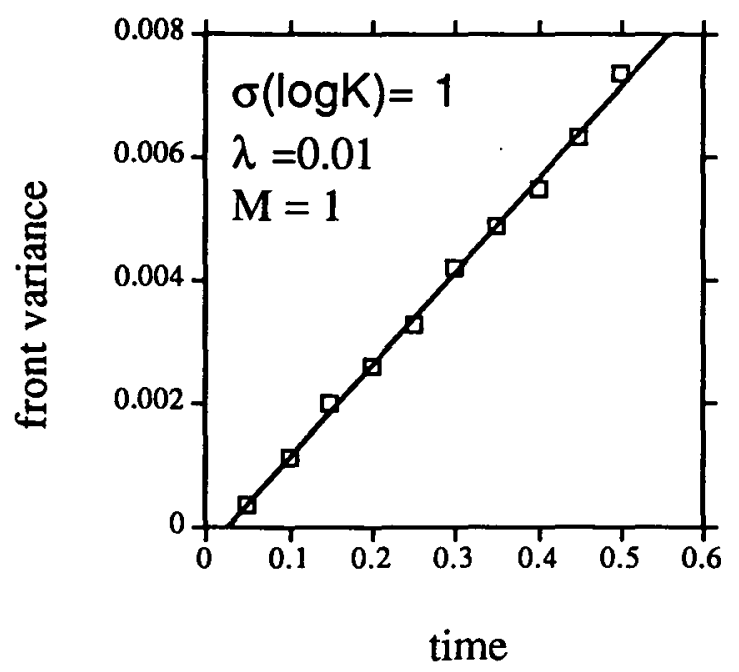

a)

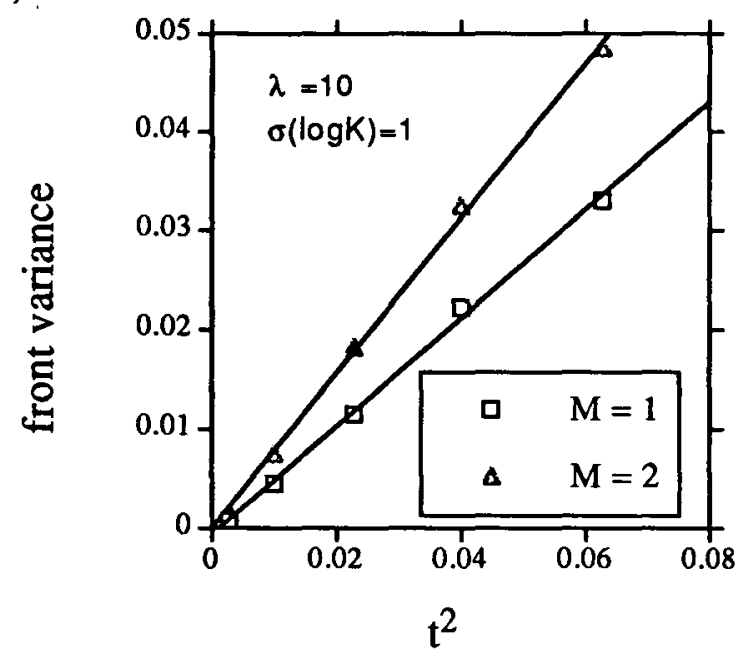

b)

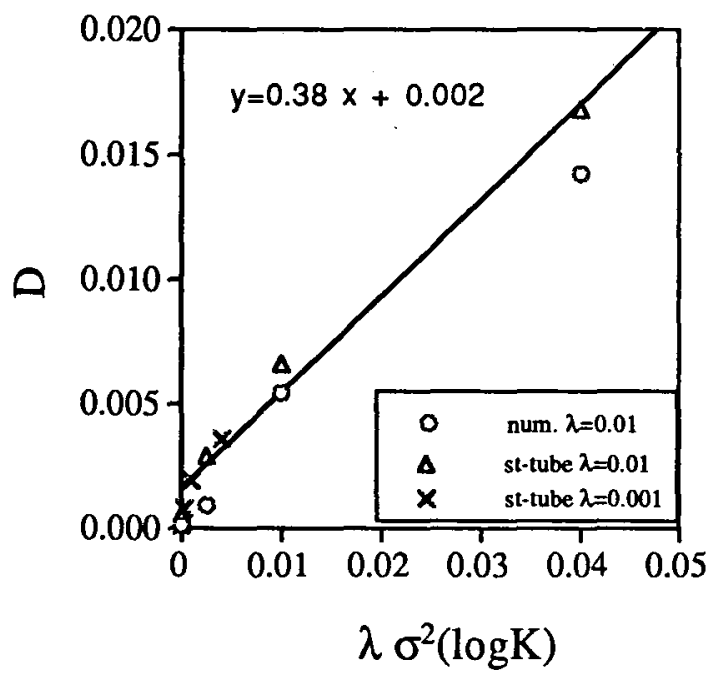

Fig. 5 - Tracer displacement. Dispersivity as a function of Gelhar's macrodispersivity.

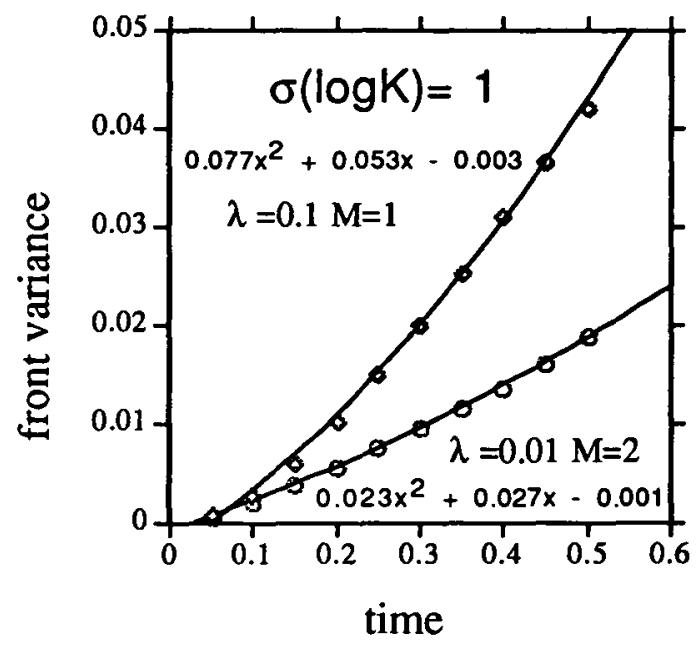

c)

Fig.4 - Front spreading as function of time

a) dispersive spreading (front variance proportional to time)

b) convective spreading (front variance proportional to time $^{2}$ )

c) mixed spreading fitted by a polynomial function $\mathrm{At}^{2}+\mathrm{Bt}+\mathrm{C}$

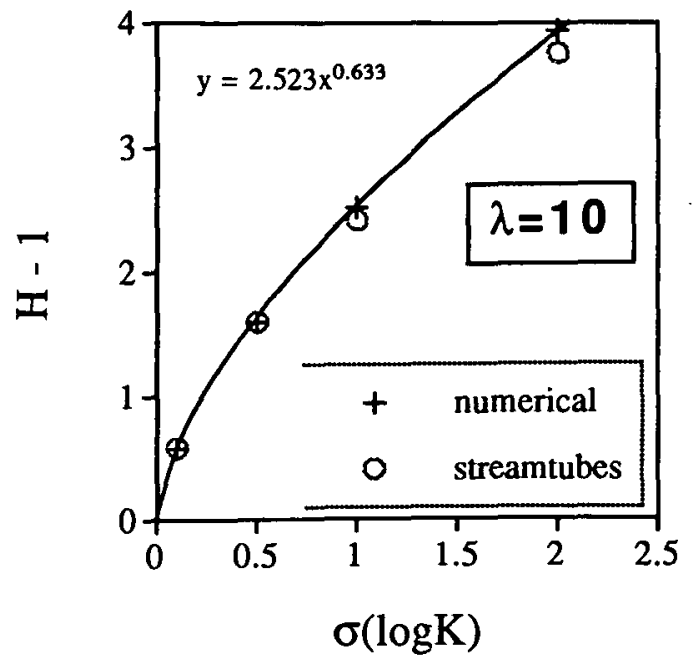

Fig. 6 - Tracer displacement through a layered medium. Heterogeneity parameter $\mathrm{H}$ as a function of permeability contrast. 

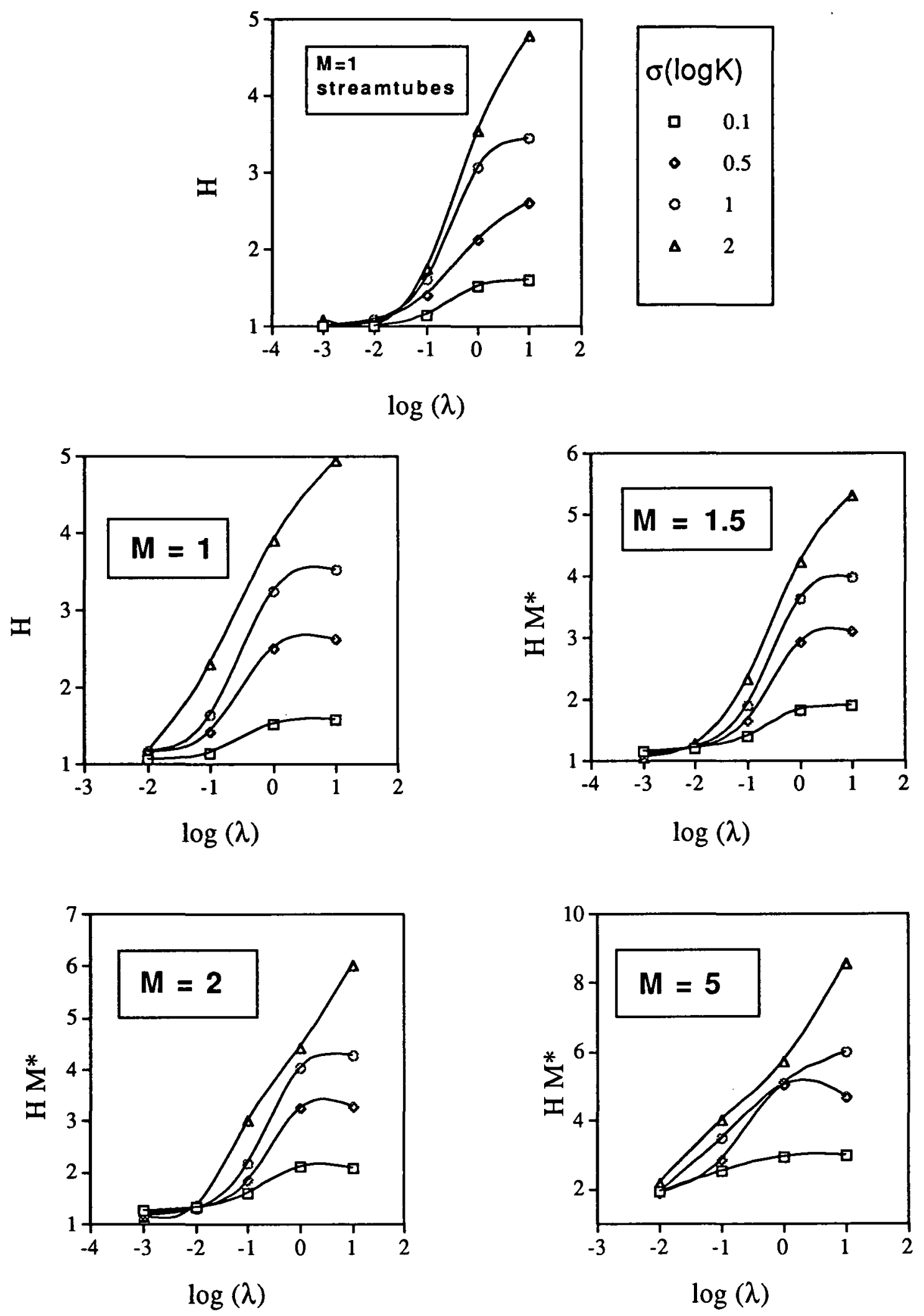

Fig 7 - Parameters $\mathrm{H}$ and $\mathrm{HM}^{*}$ as functions of correlation length for various permeability contrasts and viscosity ratios. 

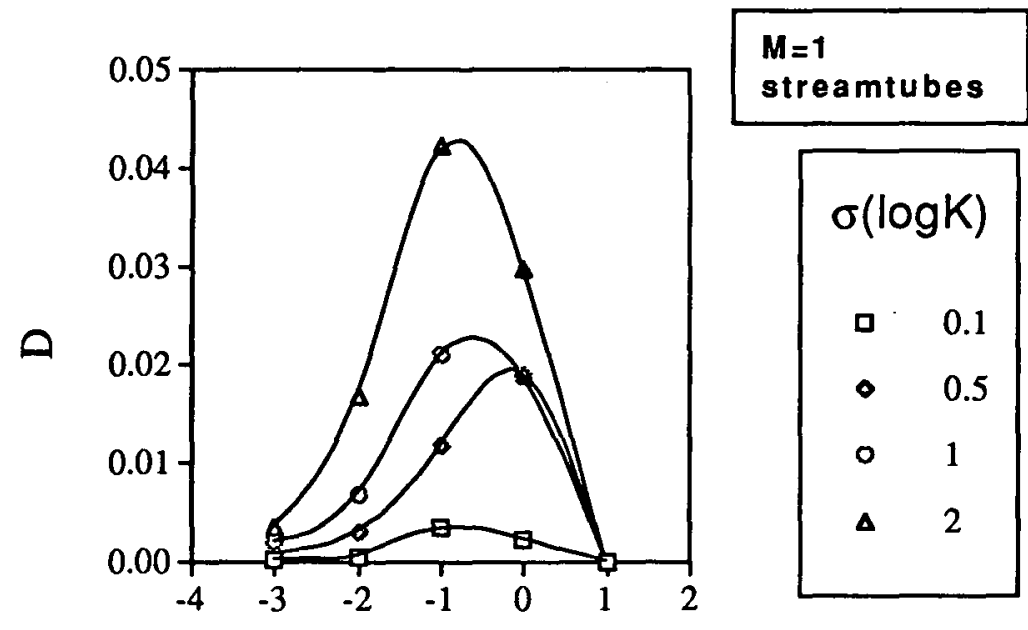

$\log (\lambda)$

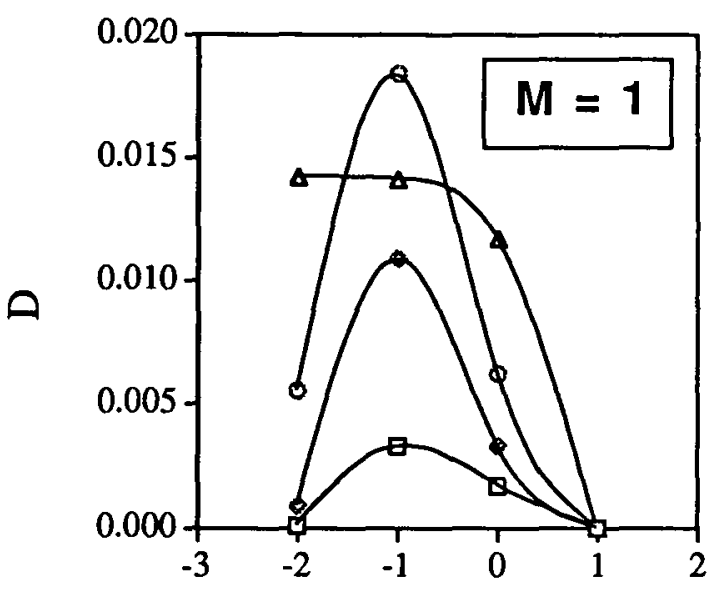

$\log (\lambda)$

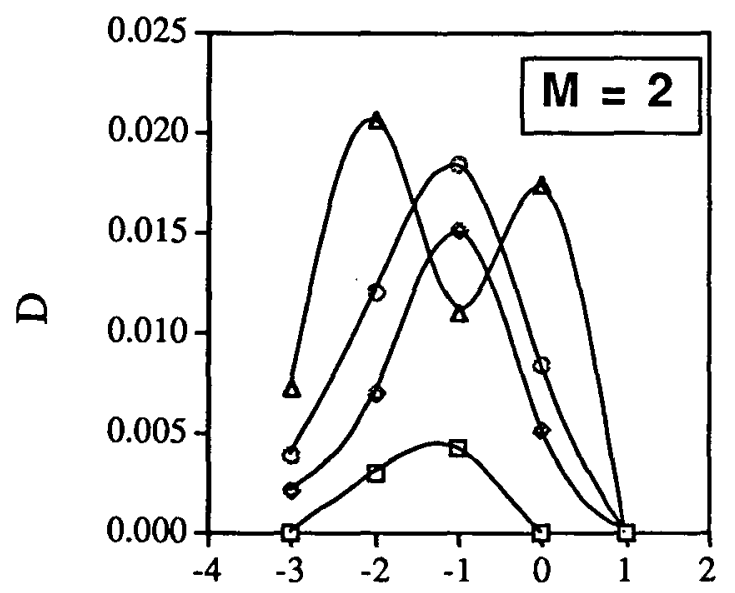

$\log (\lambda)$

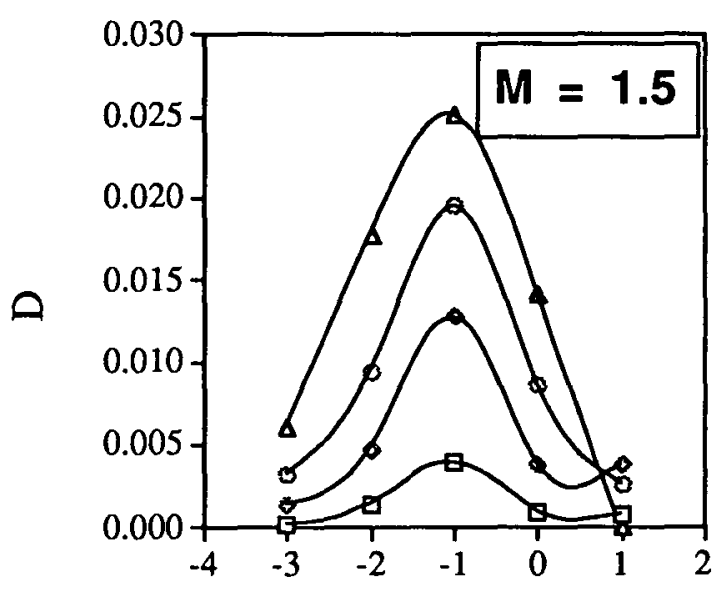

$\log (\lambda)$

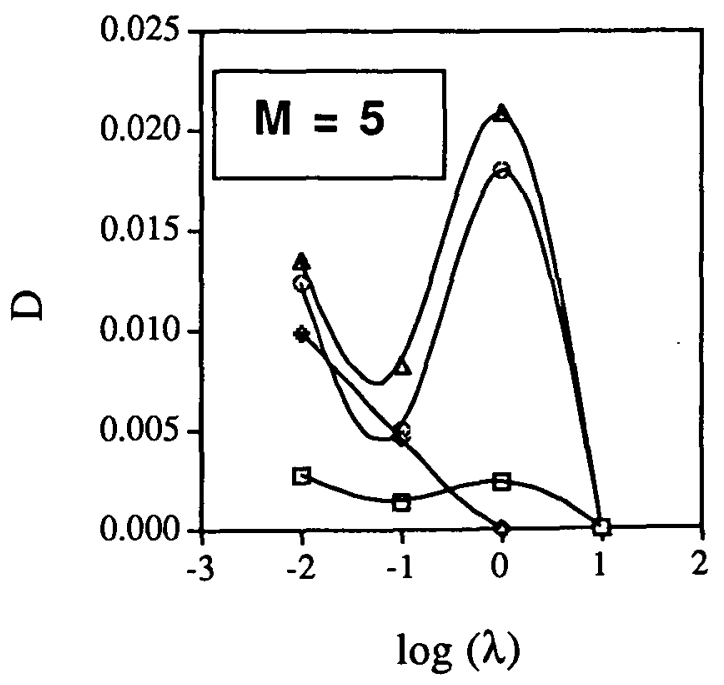

Fig. 8 - Macrodispersivity D as functions of correlation length for various permeability contrasts and viscosity ratios. 


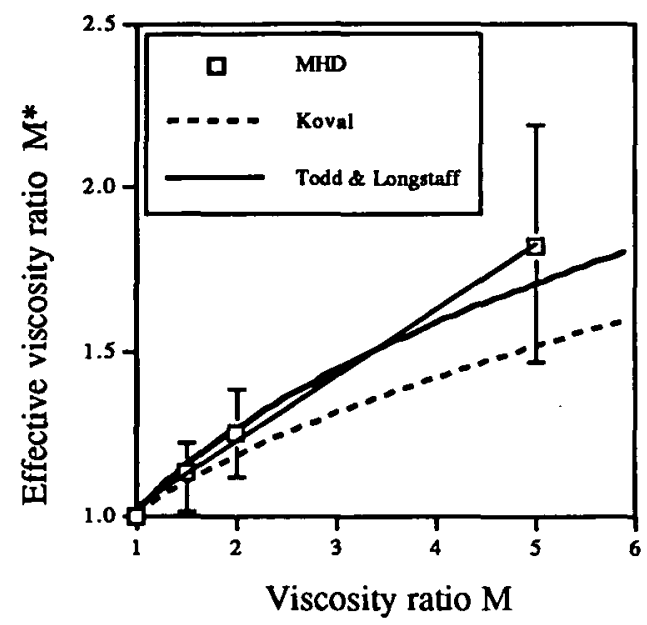

Fig. 9 - Effective viscosity ratio $M^{*}$ a function of the real viscosity ratio used in the simulation. Comparison with Koval and Todd \& Longstaff models.

The spread in the data is considerable, but there is an acceptable fit with Koval's model (Koval 1963)

$$
M^{*}=\left(0.78+0.22 M^{0.25}\right)^{4}
$$

and the more general form suggested by Todd and Longstaff (1972), using $\omega$ of the order of 0.66 ( $\omega$ is a fitting parameter in Todd and Longstaff model describing the effective mixing of the fluids).

$$
M^{*}=M^{(1-\omega)}
$$

Figure 8 shows the dispersivity, $D^{*}$, to be of the same order of magnitude for the different mobility ratio displacements. A maximum value for dispersivity (of the order of $0.02 \mathrm{~L}$ ) occurs for correlation lengths around $\lambda=0.1$.

\section{CONCLUSIONS}

We have performed numerical simulations with a low level of numerical dispersion to check the validity of the MHD equation. The MHD equation was derived by a stochastic approach and describes the 1D averaged displacement of one fluid by another in a heterogeneous medium. The following results have been derived:

1) The MHD-equation is able to model displacements that account for viscous (M) and dispersive (D) mechanisms in a heterogeneous $(\mathrm{H})$ domain.

2) The parameters $H$ and $D$ can be determined from a tracer $(M=1)$ displacement by fitting a 2 nd order polynomial to the variance of the front spreading as a function of time.
3) Displacements for $\mathbf{M}>1$ can then be predicted by using the $\mathrm{H}$ and $\mathrm{D}$ parameters determined from the tracer displacement and using an effective mobility ratio as predicted by Koval's model.

4) We observed a transition from dispersive to convective flow regimes in most cases. Yet for displacements with $1=0.01$ the flow is not purely dispersive $(\mathrm{H}>1)$, and similarly for displacements with $\mathrm{l}=10$ the flow is not purely convective.

4) For displacements with $l>=1$ (nearly layered systems) which are principally convective, the $\mathrm{H}$ parameter is related to the permeability contrast by $H=2.5 \sigma(\log K)^{0.63}$. This result has been derived for a $\log$ normal permeability distributions and may also depend on the size of the simulations $(250 \times 100)$ we used.

5) For $\mathrm{H}$ we observe a monotonic increase from 1 (dispersive) to a few units in the convective domain.

6) For a given permeability contrast, the dispersivity $D$ has a maximum around a correlation length of $\lambda=0.1$.

7) The high-order, flux-corrected finite difference simulator and streamtube simulator gave similar results for tracer $(M=1)$ displacements.

\section{ACKNOWLEDGEMENTS}

The autors ould like to thank Martin Blunt for many helpful suggestions and Mike Christie for making the finite-difference code available.

\section{REFERENCES}

Blunt, M. J. and Christie, M. A., "Exact Solutions for Viscous Fingering in Two-Phase, Three-Component Flow", SPE 22613, Proceedings of the Annual Technical Conference, Dallas, USA, (1991).

Christie, M.: "High Resolution Simulation of Unstable Flows in Porous Media," SPERE (1989) 4, No. 3, 297-303.

Deutsch, C.V. and Journel, A.G.: GSLIB Geostatistical Software Library and User's Guide, Oxford University Press (1992).

Durlofsky, L.J., "Numerical Calculation of Equivalent Grid Block Permeability Tensors for Heterogeneous Porous Media," Water Resources Research (1991) 27, No.5, 699-708.

Fayers, F.J., "An Approximate Model with Physically Interpretable Parameters for Representing Miscible Viscous Fingering," SPERE (May 1988) 3, 551--558.

Fayers, F.J., Blunt, M.J. and Christie, M.A., "Accurate Calibration of Empirical Viscous Fingering Models", Rev. Inst. Franc. Pétrole 46 (1991) 311-325.

Fayers, F.J., Blunt, M.J., and Christie, M.A., "Comparison of Empirical Viscous-Fingering Models and Their Calibration for Heterogeneoius Problems," SPERE (May 1992) 195-203.

Gelhar, L. W. and Axness, C. L., "Three-Dimensional Stochastic Analysis of Macrodispersion in Aquifers, Water Res. Res. 19 (1983) 161-180. 
Gorell, S. B., "Outlook for Calibration of Large Grid Block Models for Miscible Flooding", SPE/DOE 24186, Symposium on EOR, Tulsa, OK, USA, (1992).

King, P. R., "The Use of Renormalization for Calculating Effective Permeability ," Transport in Porous Media, (1989), 4, 37--58.

Kossack, C.A., Aasea, J.O., and Opdal, S.T., "Scaling Up Heterogeneities With Pseudofunctions," SPEFE (Sept. 1990) 226-232.

Koval, E.J., "A Method for Predicting the Performance of Unstable Miscible Displacements in Heterogeneous Media," SPEJ (June 1963)

Lenormand, R., "Transport Equations for Fluid Displacements in Heterogeneous Media: the MHD Equation", paper SPE 30797, Proceedings of the Annual Technical Conference, Dallas, (1995).

Lenormand, R.,"Determining Flow Equations from Stochastic Properties of a permeability field: the MHD Model", to appear in SPEJ, (1996).

Pickup, G. E., Ringrose, P. S., Corbett, P. W. M., Jensen, J. L., and Sorbie, K. S., "Geology, Geometry, and Effective Flow", Petroleum Geoscience, 1,37-42 , 1995

Sorbie, K. S., Feghi, F. Pickup, G. E., Ringrose, P. S., and Jensen, J. L., "Flow Regimes in Miscible Displacements in Heterogeneous Correlated Random Fields," SPE/DOE 8th Symposium on EOR, SPE/DOE 24140 (April 1992), 371-386.

Thiele, M. R., Batycky, R. P., Blunt, M. J., and Orr, F. M., "Simulating Flow in Heterogeneous Media Using Streamtubes and Streamlines," SPERE (1996) 10, No. $1,5-12$.

Todd, M. R. and Longstaff, W. J., "The Development, Testing and Application of a Numerical Simulator for Predicting Miscible Flood Performance," Trans., AIME, 253 (1972) 874--882.

\section{APPENDIX}

The purpose of this appendix is to derive the moments of the front from the MHD equation (we take $M=1$ to simplify the notation, for $M \neq 1, H$ must be replaced by the product $\mathrm{HM}^{*}$ ).

$$
\frac{\partial S}{\partial t}+u \frac{\partial f}{\partial x}=D \frac{\partial}{\partial x}\left(\frac{f}{S} \frac{\partial S}{\partial x}\right)
$$

with the fractional flow

$$
f=\frac{S}{S+(1-S) / H}
$$

The variance of the front (Eq. \$) is integrated by part

$$
\sigma_{x}^{2}=-\left[\left(x-x_{f}\right)^{2} S\right]_{0}^{L}+2 \int_{0}^{L}\left(x-x_{f}\right) S d x
$$

Before breakthrough $S(L)=0$ and $x_{5}=u t$

$$
\sigma_{x}^{2}=(u t)^{2}+2 \int_{0}^{L} x S d x-2 u t \int_{0}^{L} S d x
$$

The last integral is equal to ut

$$
\sigma_{\mathrm{x}}^{2}=-(\mathrm{ut})^{2}+2 \int_{0}^{\mathrm{L}} \mathrm{x} S \mathrm{dx}
$$

We differenciate all the terms with respect to $t$

$$
\frac{\partial \sigma_{x}^{2}}{\partial t}=-2 u^{2} t+2 \int_{0}^{L} x \frac{\partial S}{\partial t} d x
$$

Using the mass balance

$$
\frac{\partial \sigma_{x}^{2}}{\partial t}=-2 u^{2} t-2 u \int_{0}^{L} x \frac{\partial f}{\partial x} d x
$$

after integration by part

$$
\frac{\partial \sigma_{x}^{2}}{\partial t}=-2 u^{2} t-2 u[x f]_{0}^{L}+2 u \int_{0}^{L} f d x
$$

since there is no flux at exit before breakthrough

$$
\frac{\partial \sigma_{x}^{2}}{\partial t}=-2 u^{2} t+2 u \int_{0}^{L} f d x
$$

This equation is general. For the HD equation, the fractional flux is equal to

$$
f=f_{H}-d\left(\frac{f_{H}}{S} \frac{\partial S}{\partial x}\right)
$$

Consequently

$$
\frac{\partial \sigma_{x}^{2}}{\partial t}=-2 u^{2} t+2 u \int_{0}^{L} f_{H} d x-2 u d \int_{0}^{L} \frac{f_{H}}{S} \frac{\partial S}{\partial x} d x
$$

In this equation we note $C$ and $A$ the convective and dispersive contibutions to the variance. Let us first calculate the dispersive part

$$
A=-2 u d \int_{0}^{L} \frac{f_{H}}{S} \frac{\partial S}{\partial x} d x
$$

Using the variable $S$ instead of $x$ with $S(0)=1, S(L)=0$

$$
A=-2 u d \int_{1}^{0} \frac{1}{S+(1-S) / H} d S
$$

After integration for $\mathrm{H}=1, \mathrm{~A}=2 \mathrm{ud}$. For $\mathrm{H} \neq 1$

$$
A=-2 \mathrm{ud} \frac{\mathrm{H}}{\mathrm{H}-1}[\ln (\mathrm{S}(1-1 / \mathrm{H})+1 / \mathrm{H})]_{1}^{0}
$$

and

$$
A=2 \mathrm{ud} \frac{\mathrm{H}}{\mathrm{H}-1} \ln (\mathrm{H})
$$

In Equation 15, the convective contribution is

$$
C=2 u \int_{0}^{L} f_{H} d x
$$

replacing the fractional flux by its value 


$$
C=2 u \int_{0}^{L} \frac{S}{S+(1-S) / H} d x
$$

This equation is differenciated with respect to time

$$
\frac{\partial C}{\partial t}=2 \mathrm{uH} \int_{0}^{\mathrm{L}} \frac{1}{(\mathrm{~S}(\mathrm{H}-1)+1)^{2}} \frac{\partial S}{\partial t} \mathrm{dx}
$$

using the mass balance to replace saturation by flux leads to

$$
\begin{aligned}
& \frac{\partial C}{\partial t}=-2 u^{2} H \int_{0}^{L} \frac{1}{(S(H-1)+1)^{2}} \frac{\partial f_{H}}{\partial x} d x \\
& \frac{\partial C}{\partial t}=-2 u^{2} H^{2} \int_{0}^{L} \frac{1}{(S(H-1)+1)^{4}} \frac{\partial S}{\partial x} d x
\end{aligned}
$$

with $S$ as variable instead of $x$

$$
\frac{\partial C}{\partial t}=-2 u^{2} H^{2} \int_{1}^{0} \frac{d S}{(S(H-1)+1)^{4}}
$$

after integration

$$
\frac{\partial C}{\partial t}=\frac{2}{3} u^{2} \frac{\left(H^{2}+H+1\right)}{H}
$$

assuming that the integration constant is zero

$$
\mathrm{C}=\frac{2}{3} \frac{\left(\mathrm{H}^{2}+\mathrm{H}+1\right)}{\mathrm{H}} \mathrm{u}^{2} \mathrm{t}
$$

Finally, the first order time derivative of the variance is given by

$$
\frac{\partial \sigma_{x}^{2}}{\partial t}=-2 u^{2} t+\frac{2}{3} \frac{\left(H^{2}+H+1\right)}{H} u^{2} t+2 u d \frac{H}{H-1} \ln (H)
$$

After integration with respect to time

$$
\sigma_{x}^{2}=\frac{(H-1)^{2}}{3 H} u^{2} t^{2}+2 d \frac{H \ln (H)}{H-1} u t
$$

